\title{
Investigating Facebook Friendships through Five Similarity Dimensions
}

\author{
Elena - Mădălina VĂTĂMĂNESCU \\ College of Management \\ National University of Political Studies and Public Administration, Romania \\ 6 Povernei St., Sector 1, Bucharest, Romania, 010643 \\ madalina.vatamanescu@comunicare.ro
}

DOI:10.5901/mjss.2014.v5n22p252

\begin{abstract}
The unprecedented evolution of social media has led to the exploitation of the new technologies with the purpose of encouraging closer social relationships at the individual and group levels. One of the most prolific cooperation forms was objectified through the online social networks, a catalytic form of interaction that allows individuals to stay constantly connected and to exploit the complex nature of the communication devices and services. Starting from this point, the paper discusses the main forms of similarity which stand for the basis of interaction and communication among the members of Facebook social networks. The proposed approaches to similarity address the mechanisms which encourage the formation and the development of strong online social networks: the similarity as a condition, the similarity as a context, the similarity as a catalyzer, the similarity as a consequence and the similarity as a connection. The configuration of these dimensions in the context of the online social networks was tested by analyzing the case of Facebook online social networks. Each of the five dimensions was marked out from the perspective of a sociological interview-based inquiry conducted with fourteen students from a communication college. With a view to better illustrate certain findings, an online social network analysis was performed. As the preliminary results showed, Facebook, as an online social network, is used by the respondents especially for social searching and bonding social capital rather than social browsing which concerns the initiation of new contacts with unknown individuals in the direct interaction. In fact, the most important function of these communities is to allow members to discover other sources of similarity and to potentiate the similarity discovered in the offline environment through consistent communication and interaction.
\end{abstract}

Keywords: Facebook, Similarity, Online Social Networks

\section{Introduction}

The unprecedented evolution of the facilities ensured by the social media has led to the exploitation of the new technologies with the purpose of encouraging closer social relationships at the individual and group level. One of the most prolific interactions was shaped as online social networks, a form of communication that allows individuals to stay constantly connected and to exploit the complex nature of the communication devices and services.

The interest in analyzing the online social networks has grown significantly in the past years and has reunited researchers from multiple fields, leading to the development of various methods of studying the relationships among people, groups, organizations and other entities. Hereby, social relations and the relating processes within an online social network or community have become key components of human activity even though, in time, they have been limited by temporal and spatial coordinates. The respective restrictions have partially faded away due to the evolution of social media and due to their availability generalized by the use of revolutionary technologies. In particular, the emergence of the new technologies and the progress towards Web 2.0 have allowed people to organize themselves into online social networks in a manner similar to the one in which people organize themselves into social networks in the physical reality.

The differences between the early social networks and the online social networks generally consist in the mechanisms used by the members to communicate among themselves. For the communities in the physical reality, the face-to-face interaction represents the main form of interrelating, whereas for online communities the privileged place is occupied by the information and communication technologies that facilitate interaction and promote communication among individuals, anyplace and anytime. In other words, the online social networks have developed based on the hardware and software that allow individuals to share information through simple, immediate, universal, inexpensive and reliable interaction. 


\section{Literature review}

\section{Facebook facts and figures}

The term of "online (virtual) social network" refers to a Web-based service that allows individuals to build a public or semipublic profile, to articulate a list of persons with whom they share a certain connection, and to view and to explore one's own list and the list of other members from the community (Boyd \& Ellison, 2007, p. 210). At this level, there are several descriptions of the concept, depending on the perspective of the definition which often relies on a multidisciplinary approach - sociological, technological, economic, commercial-electronic etc.

Social networking sites represent a natural extension of these studies as they connect individuals in a geographically unrestrained way. On most social networking sites, individuals do not seek to meet or to establish connections with new people but, on the contrary, the prevailing intention is that of maintaining contact with the existing groups of friends and acquaintances (Boyd \& Ellison, 2007; Boyd \& Heer, 2006; Donath, 2007; Donath \& Boyd, 2004).

Facebook is a social networking site created in 2004 by a Harvard student (Mark Zuckerberg) and made available to the wide public towards the end of 2006. Its technological design allows users to add friends, send messages, update personal profiles to keep friends and acquaintances informed on any recent change, to upload and view photos. Facebook users may also create or join virtual groups, explore or develop applications, host content, find information about the interests, hobbies and statuses of other members starting from the online profiles that may be publicly or semipublicly available.

Researches on Facebook have a short history but are growing at a fast pace and are adopting, most of the times, an interdisciplinary approach. In this respect, researchers have focused upon various issues that may be organized according to several major lines: presenting the self online and managing one's image on Facebook (Boyd \& Heer, 2006; Liu, 2007; Buffardi \& Campbell, 2008; Back et al., 2010; Papacharissi, 2009; Walther et al., 2009; DeAndrea, Shaw \& Levine, 2010; Georges, 2010), confidentiality and privacy of Facebook communication (Boyd, 2008; Tufekci, 2008; Van Manen, 2010; Ledbetter et al., 2010), exploring the civic and political potential of Facebook (Smith \& Rainie, 2008; Zhang et al., 2009; Watt, 2010), the psycho-social effects generated by Facebook among teenagers (Lewis \& West, 2009; Pujazon-Zazik \& Park, 2010), the impact of the uses and gratification theory upon Facebook relating (Quan-Haase \& Young, 2010), differences among perceived audiences and real audiences (Barnes, 2006; Stutzman, 2006), relationships between teachers and students (Mazer, Murphy \& Simonds, 2007), friendships among teenagers (Lenhart \& Madden, 2007), maintaining interpersonal relationships as a maximum attraction point (Donath, 2007; Ellison, Steinfeld \& Lampe, 2007; Tufekci, 2008; Baym \& Ledbetter, 2009), the impact of social networks such as Facebook in terms of organization (Balagué \& Fayon, 2010), the construction of online identities (Tinnell, 2014; Barba, 2014; Wessels, 2012; Davis, 2012; Jin, 2013; Goode, 2010; Macek, 2013; Schandorf, 2013; De Luna, 2011; Buffardi \& Campbell, 2008; Kramer \& Winter, 2008; Walther et al., 2008, 2009 etc.

The studies on the patterns of using Facebook suggest that this is used mainly to keep in touch with offline friends and not to develop new relationships (Ellison, Steinfield \& Lampe 2007). A study involving 2000 students concluded that Facebook is used by students for reasons related to social searching that implies learning new information about persons one knows offline and much less for social browsing that refers to using Facebook to establish new contacts. Most of the students answered that they use Facebook "to stay connected with old friends or with persons they met during high-school" (Lampe, Ellison \& Steinfield, 2006, p. 168).

Ellison, Steinfield and Lampe (2007) obtained similar results indicating that students use Facebook to maintain preexisting close relationships (bonding social capital) and to stay in touch with various colleagues and acquaintances from high-school (maintaining social capital). Regarding the maintenance of social capital, Ellison, Steinfield and Lampe highlight the fact that Facebook ensures the relatively easy maintenance of contacts by the constant updating and viewing of information related to friends' activities, important events in their life etc. Concerning the maintenance of the social capital, the authors emphasize that Facebook allows users to maintain weak ties with known persons that may turn, in time, into sources of information or valuable resources. As a conclusion, it has been highlighted that Facebook fulfills an important social function that aims at facilitating the maintenance of connections with offline friends or acquaintances, both the close and the distant ones.

Facebook offers the possibility of increased social contact by a process called "friending", by means of which users create personal profiles and accumulate friends through mutual acceptance. Creating and maintaining friends has proved particularly important for young people, but in the literature there is considerable debate on the value of the often weak 
relationships existing between the members of online social networks. In this respect, Jane Lewis and Anne West (2009) conducted a study in the UK during which 16 students from the second and third year were interviewed, students that owned a Facebook account ever since Facebook was launched in all UK universities, in October 2005. According to Jane Lewis and Anne West (2009), most of the researches focused upon social networking sites are North-American and quantitative in nature. The qualitative studies of Danah Boyd $(2006,2007)$ and Sonia Livingstone (2008) represent exceptions from this perspective. The emphasis was mainly lied upon "the matrices of preexisting social contacts" (Licoppe, 2004) and upon the "culture of friending" (Boyd, 2006), so that the present study may be regarded as a step forward by its exploratory and qualitative nature.

As Boyd (2006) observed, it is difficult to evaluate the weight of these relations or the metrics used in accepting or rejecting a friendship request, therefore, the lists of friends may contain a wide range of relations starting from the closest and ending with the least close relations. At this level, the research focused upon the way in which social and personal networks are organized in clusters. For example, McCarty (2002) revealed the fact that individuals have an average of six types of clusters; these groups include family, neighbors, current job acquaintances, previous job acquaintances, friends from school and friends from the networks of other persons. On Facebook, all these categories have been initially leveled to a single one designated through the notion of "friend" (Boyd, 2006).

Nevertheless, Facebook is significantly different from other types of computer-mediated communication and from other social networking sites as their users have been and still are dependent upon the geographic distribution to a large extent and because they tend to know their friends before (Lewis \& West, 2009). Facebook allows interrelating in a variety of ways and makes up for other means of communicating with friends - offline communication, face-to-face or on the phone, supporting connections with persons that cannot be met on a regular basis and facilitating an open contact to find out relevant information on what the others in the network are doing without necessarily starting a direct communication with these. Adopting new communication technologies has raised, in time, serious problems related to their harmful effects upon the quality of interpersonal relationships. The occurrence of Facebook is no exception from this trend (Henry, 2007; Tilsner, 2008). Despite these reserves, empirical research has shown that Facebook communicational processes have healthy communicational results. Thus, Ellison, Steinfield and Lampe (2007, p. 1165) found that Facebook supports the building of social capital - "the online interactions do not divert individuals from their offline world unconditionally, but may become a support for relationships and for helping people stay in touch, even when the changes in their lives lead them far from one another". Other recent studies highlighted the fact that Facebook connects individuals despite establishing relationships on a local level or over a larger distance (Hargittai, 2007).

However, although there are numerous proofs according to which Facebook use causes positive relational results, this does not apply to all Facebook users. Certain motivations for resorting to online communication, such as social anxiety in offline contexts, may generate certain interpersonal online communication patterns that may cause harmful psycho-social results. According to Ledbetter et al. (2010), a neutral approach to Facebook communication should take into consideration both the positive, constructive results and the negative, pathological effects of using social networking sites.

\section{Similarity issues}

The issue of similarity occupies a privileged place in social sciences as many empirical studies have highlighted, in time, direct connections with other concepts of a major scientific interest: similarity - interpersonal attraction, similarity - social trust (credibility), similarity - group cohesion, similarity - conformity, similarity - social prestige, similarity - socio-economic status, similarity - persuasion etc. In this respect, researchers have systematically tried to test in various experimental contexts hypostases that may lead to the conceptual and multidisciplinary development of similarity. Whereas certain theorists have initiated new, daring hypotheses, others have chosen to reiterate previous methodological paths with a view to validating, respectively invalidating, the results obtained by their precursors.

From the perspective of cognitive psychology, similarity designates the psychological closeness or proximity between two representations - the smaller the mental distance between two concepts, the higher the similarity between them (Shepard, 1962, p. 126). Transferring the angle of analysis upon social psychology, similarity was and is defined as the high degree of correspondence between the personality, attitudes, values and interests of individuals. Moreover, experimental researches have shown that many forms of similarity are reified for determining the positive relationships among people: similarity of opinions, of interpersonal styles, of communication abilities, of socio-economic foundations, of physical aspect etc (Byrne, 1971). 


\section{Purpose and hypotheses of the study}

Within this interpretative frame, the paper aims at outlining and investigating five complementary and interconnected elements / hypostases of similarity that have the potential to turn into investigation areas for the typology of interpersonal relationships: similarity as a condition (referring to the physical and psycho-social attributes of the individual as premises for identifying similarity), similarity as a context (referring to the contextual data that support the identification and accentuation of previously identified similarity), similarity as a catalyzer (referring to the amplifying impact of perceived similarity upon interpersonal attractiveness), similarity as a consequence (referring to the similarity derived from group and intergroup processes - cohesion and conformity) and similarity as a connection (referring to the emergence of some new areas of similarity between individuals situated at a distance in terms of space and culture that share, nevertheless, common interests and preoccupations, brought to the fore by modern communication technologies).

The main hypothesis lying at the basis of the research was that the configuration of similarity among the members of a community is the expression of the areas of similarity prefigured and assumed in the offline environment. In other words, the online environment objectified in the form of the social network Facebook reiterates within emerging or existing communities the hypostases of similarities regulated by and through direct interaction, by direct interpersonal communication, by unmediated discovery and knowledge. In fact, the basic role of virtual communities from an interpersonal perspective is that of maintaining and augmenting interaction, respectively communication among those that also interrelate constantly in the offline environment, that is, within classic communities.

Operationalizing the central hypothesis on the five dimensions of similarity, the specific hypotheses may be formulated as follows:

H1. Similarity - condition: Within virtual communities, constant interaction and communication among the members relies on the similarity of interests, activities, preferences that are revealed and assumed by the individuals in the offline environment.

H2. Similarity - context: The interaction and communication among the members of a virtual community represent a projection in the online environment of the offline interrelating contexts.

H3. Similarity - catalyst: In the virtual environment, similarity (the perception of similarity) on a certain level triggers, through a hallo effect, the perception of several levels of similarity.

H4. Similarity - consequence: Within virtual communities, similarity (the perception of similarity) among individuals acts towards maintaining the group conformity and cohesion from the offline environment.

H5. Similarity - connection: Virtual communities act particularly towards maintaining or highlighting similarity with close but distant friends and not towards exploring similarity with unknown individuals.

\section{Method \\ Participants}

Fourteen students from a Romanian communication college participated to the study. The selection of students as participants to the study was determined by two main reasons: (1) to test the hypotheses by investigating the approach of the largest category of Facebook users (2) to conduct the interviews with students who initially did not have a Facebook account and were willing to make one. The participants were selected using a pre-test for indentifying student without Facebook accounts. Two main criteria of selection were followed: (1) respondents should have consented to share their account personal information and facts with the researcher during the investigation process and (2) respondents should have agreed not to talk about the investigation with their Facebook contacts. The sample comprised individuals aged between 19 and 23 ( 8 females and 6 males).

\section{Procedure}

The research aimed at investigating the main forms of similarity among the members the online social network Facebook and relied on the sociological interview-based inquiry. Using the interview in scientific research has several purposes. First of all, the exploratory purpose of identifying the variables and the relations among the variables - with the help of interviews, the information obtained being able to further guide the research upon psychological and sociological phenomena. 
Secondly, the interview may constitute the main tool for collecting data with a view to testing hypotheses. In this case, each question represents an item in the structure of the measuring tool.

The interviews took place at the communication college and were conducted during December 2013 - May 2014. Questions were posed in a relaxed informal manner so that the interview appeared more like a discussion or conversation. In order to test the formulated hypotheses, the current research was based on an intensive, thorough interview able to substantiate a pertinent qualitative research (the main criterion being the quality of the information). Each of the fourteen subjects has been interviewed twice (the criterion for selecting the moments being the period of existence of the Facebook account three months and six months after creating a Facebook account), and the interviews lasted between forty minute and one hour, intervals that allowed the highlighting of the deep structures.

In terms of the continuum freedom - rigidity, the design of the interview was characterized by a reduced number of questions, a large amount of information, complex answers, centering on the interviewed person with possibilities of repeating the meeting (Chelcea, 2004, p. 304). There were indicated the theme of the interview and several major coordinates of the formulated questions were predefined: the criteria for choosing friends on Facebook, the typologies of friends from the virtual communities supported by Facebook, the nature and frequency of communication with each typology of friends, the motivations that underlined the communicational attitudes and the effects on the relational and communicational level of Facebook interaction.

Starting from these considerations and following the typology proposed by Ghiglione and Matalon (cited in Chelcea, 2004, p. 306), the inquiry relied on a semi-directive, thorough interview, centered on the person but aiming only at one aspect, a phenomenon or an element, not the person as a whole. Also, the interviews conducted may be regarded as semi-structured, having a higher level of validity than the structured ones - approaching previously established themes and hypotheses, but allowing for the highlighting of the relevant elements, the structure of the situation and the action models. A rough interview guide was made, indicating the major issues to be approached in the discussion focusing upon the subjective experience of getting involved in the analyzed situation. For each theme included in the interview, factual and opinion questions were formulated regarding the activity of the subjects within the online social network Facebook (please see the Appendix).

The sociological interview-based inquiry was corroborated with the method of online social networks analysis, which was liable to provide a pertinent view of the configuration of similarity within virtual communities. The analysis of online social networks referred to the study of the online social structure and of its effects in terms of a set of actors (nodes) and a set of relationships that connect actors and the pairs of actors (D'Andrea, Ferri \& Grifoni, 2010, p. 14). The analysis allowed "to determine whether an online social network is closely tied, diversified or well bound, to identify its density and intensity and to study the way in which the behavior of the network members may be affected by their positions and connections" (Scott, 2000, p. 961). The objects observed were not mainly the members themselves and their attributes but the relations among members and the relational structure. The advantage of such a representation is that it facilitates the analysis of certain social processes as a product of the connections among social actors.

The collection of data consisted of three different steps (D'Andrea, Ferri \& Grifoni, 2010, p. 10). The first step consists in analyzing the connections between each actor of the virtual social network and all the other actors. The second step consists in determining certain subgroups and whether the roles that each actor plays have an impact upon the online social network. The last step resided in studying the overall characteristics of the online social network. The focus is particularly upon the size of the network, in terms of density or interconnectivity, from the perspective of the number of connections within the network as a percentage from the total of possible connections. All these steps imply that each actor from the network may answer questions about each other actor from the online social network.

Data visualization was facilitated by means of graphs with nodes and connections, and the numbers from each cell represent characteristic relations among these values, matrices, where the rows and columns designate the actors and their characteristics or hybrid approaches (that combine all the three forms mentioned) (Caschera, Ferri \& Grifoni, 2008).

An important issue was to determine the groups within complex social networks, the cliques (Ortiz-Arroyo, 2010) or clusters (Drazdilova et al., 2010). Groups are separate collectivities of individuals connected to one another by a certain type of relationship or interaction. Within a group, members have different positions - some of them occupying central positions, others remaining in the periphery and the rest being somewhere between the two categories. A group may have one or several key actors (players). Given the fact that the algorithms of clustering used today are much too complex, there began to be developed specialized software that may be able to manage efficiently the entered data, marking the areas of similarity by applying a partitioning coefficient of clusters according to similarity (Gueret, 2010, p. 286). 


\section{Findings}

The first interviews were meant to gather information about the initial friends who are included in a newly created online social network on Facebook. To this end, participants were interviewed three months after creating their Facebook account. As the respondents reported, the average number of friends was 112 while for 12 out of 14 participants, most of their friends were of the same gender. For example, Andrei (21 years old) had 106 friends, most of them (91) males. When asked if gender distribution was random or if there were any clear reasons for this configuration, the answer was - "I have introduced in my Facebook list my best friends, those that I get along with very well, that I go out and have fun with and that are, obviously, boys. The girls from Facebook are friends and acquaintances that I like for various reasons, school mates, friends from high-school etc". The reiteration of the question in the second interview - that lasted for almost fifty minutes (six months after the account had been created) - highlighted a rather similar situation. Andrei had 182 friends on Facebook, 142 boys and the rest of them girls. Mentioning the topic of his friends' gender distribution followed the same lines - "It is simple to explain why most of my friends are boys - it is with them that I have the most things in common, they are my bodies, those that I go to parties with, with whom I go and have a beer, see a movie, I feel good in their company and I am interested in staying in touch with them on Facebook if there is not enough time to talk when we meet or when there are strangers around. Just a few girls from my list are truly my friends and we go out together, the others are colleagues from the faculty or from the NGOs that I have collaborated with over the course of time, girls that I flirted with online because I did not want to approach them directly, ex girlfriends ...".

The same situation was observed in Ana's (19 years old) case. At the time of the first interview, she mainly had girl friends (68 out of 117), the argument being that "girls are for friending, boys are for flirting, and too many boys would become a burden". The rapport between girl and boy friends was quite similar when the second interview occurred, although the total number of friends increased to 173 . As previously mentioned only two respondents reported a different situation, but the common pattern was that both male participants in the study were activists in a non-governmental organization with predominantly female members who were included ab initio in their personal Facebook network.

To sum up, the results show that the similarity as a condition occupies a very important part in choosing friends and in including them into personal online networks. The subjects consider from the start that they have most things in common with friends of the same gender, that they may have the most interesting and genuine communication and interrelation with the correspondent gender.

Analogously, the next questions highlighted the relevance of the age and of the socio-professional status within the similarity as a condition framework. As all the respondents mentioned, most of their friends had ages between 19 and 24 , and were students. If during the first three months of the Facebook account, there were few friends outside this age interval, at the time of the second interview, their number increased but in an insignificant manner. According to the subjects' statements, these new friends have been met during volunteering projects, among the university tutors or among acquaintances from other specialized online social networks. It is worth remarking, at this level, the fact that the respondents' attention was focused firstly on friends with similar ages and with the same socio-professional status, the basic premise being the fact that the persons in this category are also those that they share the most common interests with. In other words, the similarity as a condition dimension included a forth element, the similarity of interests.

Shifting the attention towards similarity as a catalyzer, the investigation proceeded with the analysis of the perceived similarity which potentiates interpersonal connections by enhancing interpersonal attraction. At this point, the following interview questions were designed to address this perspective on similarity. Thus, the fifth item from the interview guide aimed at highlighting the subject's interests and hobbies.

Focusing on Oana's example (22 years old) which is majorly indicative of the other cases, several coordinates may be traced. Within the first interview, Oana mentioned three major areas of interest - urban art, public relations and organizational culture - and two main hobbies - music and films. At the second interview (conducted three months later), the hobbies remained the same whereas the interests had been redefined - urban art, volunteering and public relations. Although the register was largely the same, the fact that organizational culture no longer constituted for Oana a primary field occasioned a novel approach within the second interview. The question was whether there was any link between shifting her interests and the communication with friends who subscribed to the previous area of interest (organizational culture). In this regard, a cogent aspect was underlined - a change in interests (namely a change at the similarity as a condition level) brought about a change in the permanence and consistency of communication with friends sharing the respective interest. Oana claimed that she had gradually stopped initiating conversations with her group of friends who were focused on the organizational culture dynamics, giving more and more time to discussions with the volunteers she 
was collaborating with. In other words, a directly proportional relation between the importance given to a certain interest and the communication with the associated individuals may be observed at this point.

For the sixth question - "On what criteria do you initiate a friend request on Facebook?", the subjects' answers were evocative of the similarity dimensions, to the extent in which all the five hypostases of similarity have been highlighted. Almost in every case, the initiation of friendship requests was mainly done in the first three months and was aimed at the offline friends that the subjects wanted to have as Facebook friends and communication partners. It happened similarly with the friendship requests - these came from offline close friends, from faculty colleagues, former high-school colleagues and friends from the native towns. After the first three months, the main form of including new friends into the Facebook list was accepting friendship requests from friends met during new activities conducted in the offline environment and from persons unknown from direct interaction, but who shared the same interests and hobbies. Participants also initiated friendship requests with a view to establishing Facebook friendship with some of the friends of their friends, met or heard of during direct encounters. For example, as Alina (21 years old) mentioned. "my community especially gathers my dearest friends and then their dearest friends and so on. Of course, as I have been meeting new people during the past three months, I considered it natural to make them part of my online network. It is easier to develop our relations both virtually and physically". Also, Razvan (22 years old) added that "in the past months, I initiated and accepted friendship requests to and from some of my team mates. Since I joined a handball team two months ago, I have been interested in discovering more about the other players and the best way to do it was searching through their Facebook profiles". At his turn, Andrei voiced a common pattern among the respondents, namely the inclusion of friends from other online specialized networks into their virtual communities - "as the new faces are concerned, most of them are poker players with whom I use to play on Poker Stars platform. I am mainly interested in knowing better the winners of different tournaments, in finding out more about their lives and hobbies by searching through their Facebook profiles".

Synthesizing, the architecture of the personal online social networks relied to a great extent on that of the classic communities. The members of these networks are generally the subjects' offline friends, selected according to criteria derived from the similarity as a condition dimension (age, gender, socio-professional status, interests, activities etc). Then, similarity as a context becomes a milestone for the network reinforcement as the individuals' attendance to certain formal contexts (the faculty, a NGO etc), respectively informal (the neighborhood, volunteering, hobbies) redirects their attention to the novel acquaintances. Each thematic event or interactional frame puts similar people into contact, potentiating their interest to intensify their communication and the need to expand the interaction in the virtual world as well.

The process of conferring the quality of a Facebook friend to persons that are unknown directly but share the same passion for online poker reveals both the role of similarity as a catalyzer and that of similarity as a connection. The virtual environment facilitates the creation of certain links among persons from other cultures and of other nationalities but based on a common interest that is relevant for both parties. The common hobby involves the perception of a new dimension of similarity so that including the game partner from a specialized online network looks like a natural thing to do. Not least, the fact that the interviewed students chose to include in his Facebook list friends of friends highlighted the issue of the similarity as a consequence, a form of consolidating the links with the group of belonging with a view to reinforce the cohesion by multiplying and developing the relations among its members.

In order to relieve the features of the personal online social networks, the seventh question - "What category do most of your friends fall into?" - addressed the configuration of the respondents' communities. At this level, Andrei's example is indicative of the overall cases. If after the first three months the Facebook list of friends contained only the friends that Andrei interacted with directly and frequently, and close friends that were far away (in the native town, in other universities from Romania or from around the world), during the second interview there were also mentioned the category of new friends that the subject tried to get to know better through Facebook and the category of friends that were met only virtually. However, though two new categories of friends appeared, the previous categories prevailed in terms of the friends number, most of Andrei's Facebook friends remaining the ones with whom he interacts directly and regularly (close childhood friends that are still in Bucharest, faculty mates that the subject has closer relationships with etc). The motivation behind this configuration is expressed clearly by the subject - "I want to be surrounded on Facebook by the closest people in my life, by my most important friends. In fact, this is the role of Facebook, to give us the possibility to stay in touch when we cannot meet or when we want to announce something important without having to make a hundred phones calls. The event is announced and who is in favor answers by attending the party or any other type of activity. The signals on Facebook are $90 \%$ for those that we can meet with anytime, although I don't mind if distant friends or friends that are too busy may also find out the news". 
To gain a more pertinent perspective upon the main clusters from the virtual community of Andrei, the method of online social networks analysis was used, highlighting several cogent aspects: the dimension and the nature of subgroups within the community, the direct friends (marked with 0 ) versus the friends of friends (marked with 1 ), the connections among the direct friends and the friends accepted by Andrei within his community or through direct friends (marked with red interrupted arrows), the numerical hierarchy of friends and the evolution of the network from the first to the second interview.

Analyzing the architecture of Andrei's community from the two moments we may notice, first of all, the evolution of the number of clusters from eight to eleven (there are three new clusters, respectively the cluster formed of the friends from the specialized online network Poker Stars, of the NGOs collaborators and of the university tutors). Secondly, by reorienting the interest towards other areas of activity (volunteering and online poker), the clusters associated with previous interests have remained the same in terms of numbers (see the groups of friends met during activities dedicated to extreme sports and marketing). Thirdly, two clusters have experienced a significant evolution - the group of faculty colleagues (from 37 to 89 members) and the group of current neighbors (from 5 to 16 members). The explanation is simple since during the three months interval between the two interviews, Andrei had the necessary time to make new offline friends at the faculty and also in the area where he lived in Bucharest. These friends have been subsequently integrated in his virtual community from the Facebook network. Not least, it should be noticed the fact that the number of friends of a secondary level (friends of friends), assumed by Andrei as his own friends grew five times in three months (from 7 to 35 friends). This evolution may be explained from the perspective of the similarity as a catalyzer and of the similarity as a consequence - meeting directly some of his friends' friends has allowed Andrei to discover certain areas of similarity that have turned into the basis for new connections both in the offline and the online environment. Thus, the expansion of the social capital is the result of a process of direct knowledge and assumption of the similarity discovered within virtual interrelating (as seen in the table and figures below).

Table 1. Summarization of the number of friends by categories

Typology of friends

Faculty colleagues

High-school colleagues

Current neighbors

Native town neighbors

Sport friends

Public relations friends

Marketing Friends

Online Poker friends

University tutors

NGOs collaborators

Ex-girlfriends

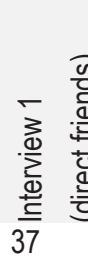

26

5

15

5

5

4

$-$

Total

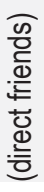

西

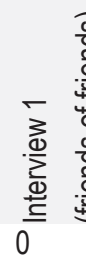

0

0

0

3

3

1

$-$

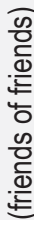

37

5

15

27

$5 \quad 11$

$15 \quad 15$

8

8

5$$
-
$$$$
-
$$

- 3

$2 \quad 2$
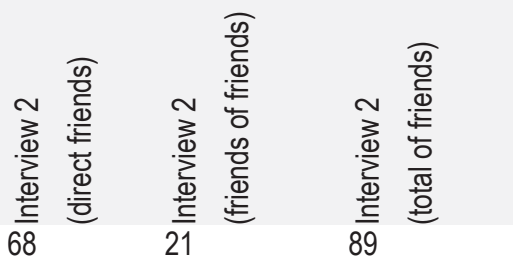

27

16

17

8

8

5

5

2

3

2

$\begin{array}{llll}106 & 147 & 35 & 182\end{array}$




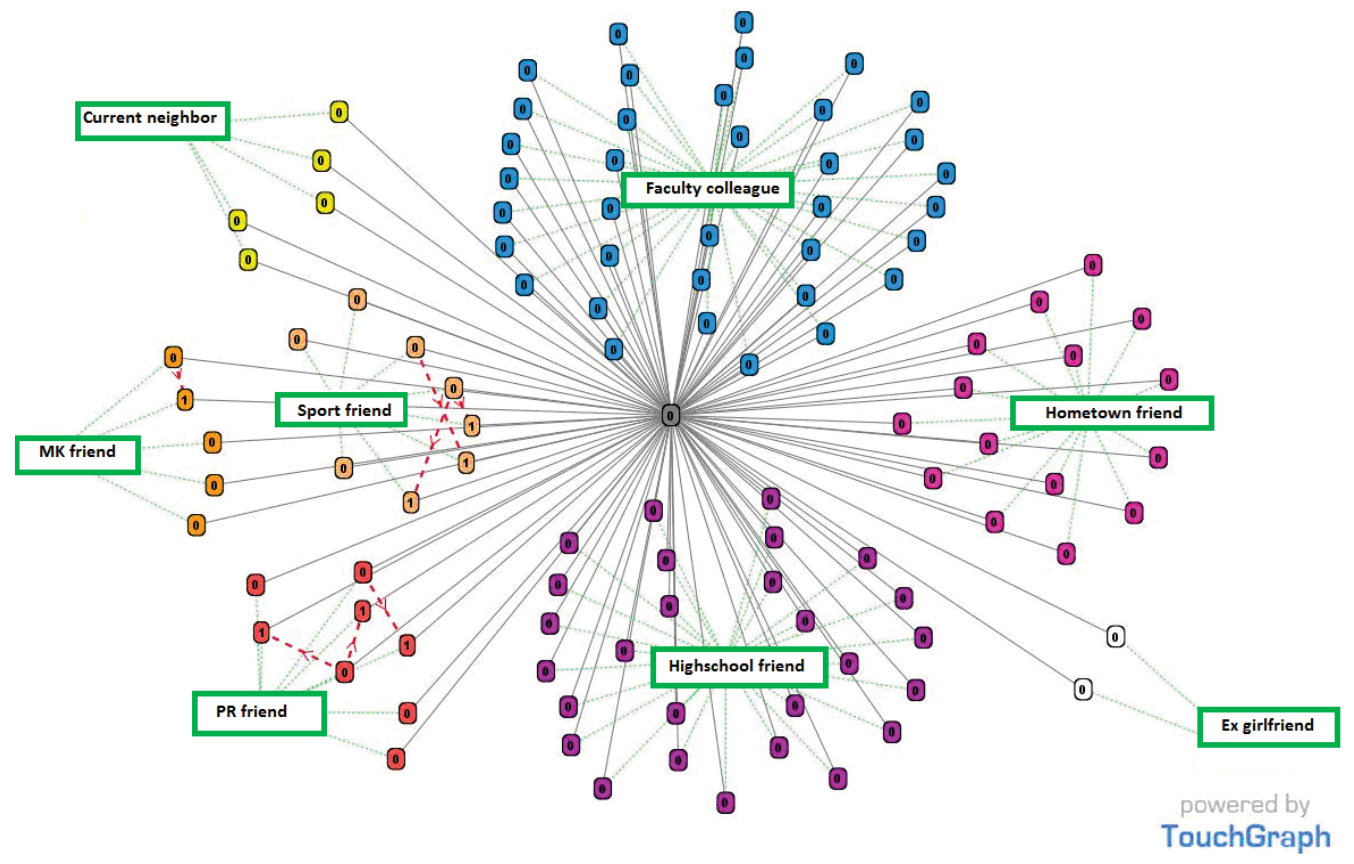

Figure 1. The architecture of the online social network at the moment of the first interview

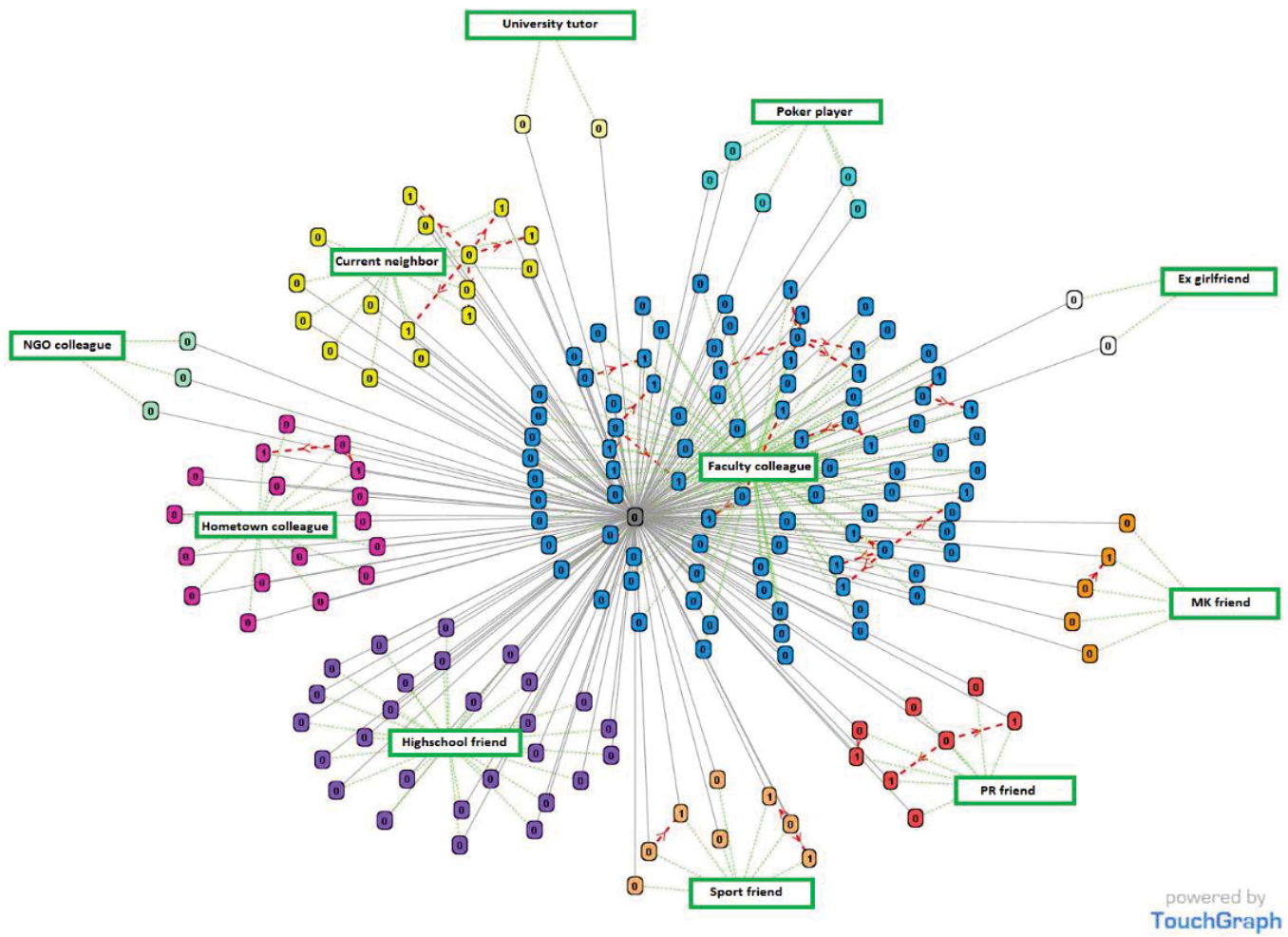

Figure 2. The architecture of the online social network at the moment of the second interview 
The eighth question - "Which of your friends do you communicate with most frequently and for the longest time on Facebook?" - also highlighted the categories of friends that prevail in terms of number of individuals. In almost all cases, the participants in the study communicate most frequently with the close friends they interact with directly on a regular basis. In terms of duration, they communicate most with old friends that are away for a certain period of time. The conversation topics with the first category rely on discussing about opinions and the activities that they took part in together (meetings, parties, conferences etc), about the persons they know, about faculty related issues etc, whereas discussions with the second category of friends focus on sharing experiences, soul problems, major dilemmas. At this level, it is revealed the fact that friendships established in the distant past, based either on the similarity as a condition or on the similarity as a context are those that prevail from the communicational perspective. Such relationships have a particular essence that is extrapolated from the offline environment towards the online social networks. As Mara (19 years old) mentioned, "rarely have I talked about my deep feelings with new friends from Facebook. There are things which cannot be shared with others, except for my childhood bodies.". Consistent with this perspective, Radu (22 years old) posited that "the time spent with my Facebook friends depends on the issues to be debated. Sometimes, we have school tasks to discuss, other times, we plan spare time activities and hangouts. Still, I use to spend more time solving heart dilemmas with my best pals from highschool.".

The next question - "What are, according to you, the advantages of Facebook communication as compared to communication on other online social networks?" - took into consideration both the synchronous communication by means of the live chat and the asynchronous communication by means of the comments posted on the personal wall or on that of friends. From this perspective, Oana mentioned several aspects that place communication on Facebook in front of the communication from other online social networks: first of all, Facebook provides simultaneously the opportunity of communicating privately and publicly according to the particularities of the situation; secondly, friends may express their opinions regarding numerous aspects that may be seen by the entire community - from commenting upon photos or status updates to endless wall messages; not least, on Facebook everything communicates - the photos, the comments, the information available on the user's profile etc.

From a bird's-eye view, it should be mentioned that the answers provided by respondents to the eighth and the ninth questions within the two interviews have reflected the same perception upon reality, the interview conducted after six months reiterating the opinions presented three months earlier. Moreover, due to the similarity of answers given by the subject during the two interviews and the absence of future mutations, the presentation of the statements on succeeding items will be done in a corroborated and not comparative manner.

Most of the participants' answers to the tenth question - "Did you ever join a group because your friends were already part of the respective group?" were negative, but additional questions revealed the fact that subjects preferred to initiate certain individualized contacts with persons from their friends' groups, with the so-called friends of friends who, for various reasons, looked similar. As Andre stated, 35 from his Facebook list of friends were, in fact, acquaintances with whom he interacted directly as friends of his friends and to whom the subject sent friendship requests with a view to establishing unmediated connections. In this respect, the similarity as a consequence was a result of the similarity as a catalyzer. In other words, Andrei extended the similarity assumed in the relationship with his friends to his friends' friends also.

The eleventh question - "Do you consider that Facebook helps you in developing your relationships with a certain category of friends?" - highlighted once again the relevance of the similarity as a catalyzer. Thus, according to Mara, Facebook helps particularly in developing relationships with faculty colleagues due to the opportunity of discussing upon various subjects that are not related to the faculty, topics meant to facilitate mutual discovery and knowledge that may also highlight other similar dimensions in terms of personality, attitudes, behaviors, beliefs etc. Also, Facebook helps in developing relationships with friends met through certain specialized online networks such as poker sites (Poker Stars, Full Tilt Poker), by sharing experience or by discussing post-factum upon certain situations experienced during the games, according to Andrei's point of view. On the contrary, Facebook has no major impact upon developing relationships with childhood friends that are either far away or close. In this case, the role of Facebook is to maintain the communication channel open, the friendship connections being already closely tied.

The twelfth question - "What determines you to include in your list of friends a person that you have not yet met by direct interaction" - resumed, tangentially, certain aspects assumed by the preceding items, confirming the importance of similarity as a connection understood as similarity as a condition objectified in the virtual environment. The link between the interviewed students and persons unknown directly but that appear in their Facebook list was represented by the common passions, hobbies and interests. Hereby, a shared preference, relevant for both parties, constitutes a necessary 
and sufficient condition to bind a relation of friendship in the online environment, although, as Radu admits, only a small part of his Facebook friends are included in this category.

Analogously, the thirteenth question - "What determines you to communicate with this person frequently although you have not yet had the chance of meeting him directly?" - gradates the overall problem. According to Andrei, communication with these friends relies on their common interest or passion - "The communication with friends that he plays online poker with and that refers to the experience lived and acquired during the games is essential. If you know how to make use of such information, you may become better at what you like". Therefore, obtaining useful, often captivating information from more experienced persons becomes a motivating factor for the constant initiation of communication, the similarity as a connection based on sharing passions ensuring the coherence of interaction.

To a certain extent, friendships among individuals both in the offline and the online environment do not rely on a summation of the similarity areas, but on the degree of relevance attached to each area by each subject. The common interest in or passion for certain fields of activity may be only occasionally more important than the similarity of age, social gender, socioprofessional status. Also, the desire to avoid conflicts and, implicitly, to preserve the groups' approbation and validation (similarity as a consequence) may act as a suppressive factor in accepting friendships with a conflicting potential.

The last question - "What would be, according to you, the profile of a friend impossible to accept on Facebook" - completes the perspectives proposed by the previous items. As a common pattern, most of the subjects described the impossible friend to accept as opposite to themselves (without being aware of this). At this level, Andrei's response is indicative of the general approach on this matter - "The friend that is impossible to accept is somebody I have nothing in common with, that attacks unconditionally and is never satisfied by anyone". In other words, an individual that he would not accept on Facebook is a person with whom he shares no characteristic from the similarity as condition and similarity as a context dimensions, a person that would elude any principle of the similarity as a consequence (social validation, cohesion, group conformity), a person that would act as an obstacle for the emergence of the similarity as a catalyzer.

\section{Discussion and conclusions}

Investigating the Facebook online social networks has revealed the configuration of the various hypostases of similarity in the virtual environment. Moreover, the answers to the interview questions validated the main hypothesis and the five secondary hypotheses of the research. According to the findings, the emerging or existing communities on Facebook reiterate the design of the existing similarity from the physical communities, maintaining and enhancing interaction, respectively the communication among those that constantly interact within the offline environment.

Focusing upon the relations established in the offline environment, through direct interaction, the main dimensions updated in the online environment are also those influencing or determining the close connections from the physical reality: similarity as a condition, similarity as a context, similarity as a catalyzer and similarity as a consequence. All these elements are also extrapolated within virtual communities, the latter projecting and amplifying the similarity among individuals through communication, social co-presence, mutual exposal, sharing.

In this respect, a new concept may be advanced in order to explain the constitutive and functional basics of the Facebook online social networks - the concept of "projected communities". The projected communities are ambivalent in nature, as they represent a projection of the offline social networks in the virtual environment, and, at the same time, they stand for a individual project, as he is fully free to include or exclude friends in and from his virtual group.

It should be noted that, on this level, the only hypostasis of similarity, organically associated with the opportunities offered by the virtual environment (similarity as a connection) does not occupy a crucial place in expanding the social capital from the online social networks. The items focused upon the testing of the similarity as a connection have revealed the fact that this does not constitute an important factor of forming connections and of interrelating in the online environment, on the contrary, in an eventual hierarchy of elements, the similarity as a connection would occupy the last position in terms of the relevance or of the degree of impact upon the crystallization of friendships. Generally, the subjects are only slightly interested in initiating contacts and establishing new relationships on Facebook, their attention being focused upon maintaining and developing the relationships that have already been established based on such criteria as: the similarity of socio-professional statuses, of the socio-economic environments, of opinions, beliefs, activities, formal and informal contexts etc.

In this light, the similarity as a connection is invested with signification, with the meaning of similarity as a condition unaffected by spatial restrictions. According to the results of the research, subjects accepted friendship requests or initiated 
friendship requests after having viewed the information from the profile of a certain person, after having analyzed the basic information (for example, age, social gender, address) and the information referring to interests and preferences, education and job etc. Also, including an unknown individual directly in one's online network was the result of sharing certain common interests, highly relevant for both parties (the status of member in certain specialized online social networks) or common hobbies (the passion for interactive games, for example). At this level, the similarity as a condition is outweighed by the similarity as a catalyzer that multiplies and amplifies the initial areas of similarity.

In conclusion, as a social networking site, Facebook is particularly used for reasons related to social documentation (social searching) and maintaining preexisting close relationships (bonding social capital) that imply knowledge of new information about persons met offline, and less for social exploration (social browsing) that refers to using Facebook to initiate new contacts. Moreover, the most important contribution of this online social network is related to two central dimensions: the opportunity of developing the relationships crystallized in the offline environment in multivalent ways and of maintaining the communication channels open with friends from the offline environment that are away or that, due to limited time, do not have the possibility to interact directly constantly.

Acknowledgement: This work was possible with the financial support of the Sectoral Operational Programme for Human Resources Development 2007-2013, co-financed by the European Social Fund, under the project number POSDRU/159/1.5/S/134650 with the title "Doctoral and Postdoctoral Fellowships for young researchers in the fields Political, Administrative Sciences, Communication Sciences and Sociology".

\section{References}

Back, M. D., Stopfer, J. M., Vazire, S., Gaddis, S., Schmukle, S. C., Egloff, B., \& Gosling, S. D. (2010). Facebook Profiles Reflect Actual Personality, Not Self-Idealization. Psychological Science, 21(3), 372 - 374.

Barba, E. (2014). Toward a language of mixed reality in the continuity style. Convergence: The International Journal of Research into New Media Technologies, 20(1), 41-54.

Balagué, C., \& Fayon, D. (2010). Facebook, Twitter et les autres... Intégrer les réseaux sociaux dans une stratégie d'entreprise. Paris: Pearson Education France.

Barnes, S. B. (2006). A Privacy Paradox: Social Networking the United States', First Monday 11(9). Available at http://www.firstmonday.org/issues/issue11_9/barnes/index.html.

Baym, N. K., \& Ledbetter, A.M. (2009). Tunes that bind? Predicting friendship strength in a music-based social network. Information, Communication \& Society, 12, 408-427.

Boyd, D. (2006). Friends, Friendsters and MySpace Top 8: Writing Community into Being on Social Network Sites. First Monday, 11(12). Available at http://www.firstmonday.org/issues/issue11_12/boyd/.

Boyd, D., \& Heer, J. (2006). Profiles as Conversation: Networked Identity Performance on Friendster. In Proceedings of Thirty-Ninth Hawai'i International Conference on System Sciences (pp. 59-69). Los Alamitos, CA: IEEE Press.

Boyd, D., Ellison, \& N. B. (2007). Social Network Sites: Definition, History and Scholarship. Journal of Computer-Mediated Communication, 13(1). Available at http://jcmc.indiana.edu/vol13/issue1/boyd.ellison.html.

Boyd, D. (2008). Facebook's Privacy Trainwreck. Exposure, Invasion, and Social Convergence. The International Journal of Research into New Media Technologies, 14(1), 13-20.

Buffardi, L. E., \& Campbell, W. K. (2008). Narcissism and Social Networking Web Sites. Personality and Social Psychology Bulletin, 34(10), $1303-1314$.

Byrne, D. (1971). The attraction paradigm. New York: Academic Press.

Caschera, M. C., Ferri, F., \& Grifoni, P. (2008). SIM: a dynamic multidimensional visualization method for social networks. PsychNology Journal, 6(3), 291-320.

Chelcea, S. (2004). Metodologia cercetării sociologice. Metode cantitative şi calitative, Second edition revised and expanded. Bucharest: Editura Economică. 
Chin, A., \& Chignell, M. (2010). DISSECT: Data-Intensive Socially Similar Evolving Community Tracker. In A. Abraham, A. Hassanien and V. Snasel (Eds.), Computational Social Network Analysis Trends, Tools and Research Advances (pp. 81105). Springer-Verlag London Limited 2010.

D’Andrea, A., Ferri, F., \& Grifoni, P. (2010). An Overview of Methods for Virtual Social Networks Analysis. In A. Abraham, A. Hassanien and V. Snasel (Eds.). Computational Social Network Analysis Trends, Tools and Research Advances (pp. 126). Springer-Verlag London Limited 2010.

Davis, K. (2012). Tensions of identity in a networked era: Young people's perspectives on the risks and rewards of online self-expression. New Media \& Society, 14(4), 634-651.

DeAndrea, D. C., Shaw, A. S., \& Levine, T. R. (2010). Online Language: The Role of Culture in Self-Expression and SelfConstrual on Facebook. Journal of Language and Social Psychology, XX(X), $1-18$.

De Luna, T.P. (2011). Performing the Face. A look at the embodied sexuality in the profile photos of selected Facebook users. 2011 International Conference on Social Science and Humanity. IPEDR 5. Singapore: IACSIT Press.

Donath, J., \& Boyd, D. (2004). Public Displays of Connection. BT Technology Journal, 22(4), 71-82.

Donath, J. (2007). Signals in Social Supernets. Journal of Computer-Mediated Communication 13(1). Available at http://jicmc.indiana.edu/vol13/issue1/donath.html.

Drazdilova, P., Obadi, G., Slaninova, K., Martinovic, \& J., Snasel, V. (2010). Analysis and Visualization of Relations in eLearning. In A. Abraham, A. Hassanien and V. Snasel (Eds.), Computational Social Network Analysis Trends, Tools and Research Advances (pp. 291-318). Springer-Verlag London Limited 2010.

Ellison, N. B., Steinfield, C., \& Lampe, C. (2007). The Benefits of Facebook 'Friends:' Social Capital and College Students' Use of Online Social Network Sites. Journal of Computer-Mediated Communication, 12(4). Available at http://jcmc.indiana.edu/vol12/issue4/ellison.html.

Georges, F. (2010). Identités Virtuelles. Les profils utilisateur du web 2.0. Paris: CPI France Quercy - Mercuès.

Goode, J. (2010). The digital identity divide: how technology knowledge impacts college students. New Media \& Society, 12(3), 497-513.

Gueret, C. (2010). Nature-Inspired Dissemination of Information in P2P Networks. In A. Abraham, A. Hassanien and V. Snasel (Eds.). Computational Social Network Analysis Trends, Tools and Research Advances (pp. 267-290). SpringerVerlag London Limited 2010.

Hargittai, E. (2007). Whose Space? Differences among Users and Non-users of Social Network Sites, Journal of ComputerMediated Communication, 13(1). Available at http://jcmc.indiana.edu/vol13/issue1/hargittai.html.

Jin, S. A. (2013). Peeling back the multiple layers of Twitter's private disclosure onion: The roles of virtual identity discrepancy and personality traits in communication privacy management on Twitter. New Media \& Society, 15(6), 813-833.

Kramer, N., \& Winter, S. (2008). Impression management 2.0: The relationship of self-esteem, extraversion, self-efficacy, and self-presentation within social networking sites. Journal of Media Psychology, 20, 106-16.

Lampe, C., Ellison, N., \& Steinfield, C. (2006). A Face(book) in the crowd: Social searching vs. social browsing. Proceedings of the 2006 20th Anniversary Conference on Computer Supported Cooperative Work (pp. 167-170). New York: ACM Press.

Ledbetter, A. M., Mazer, J. P., DeGroot, J. M., Meyer, K. R., Mao, Y., \& Swafford, B. (2010). Attitudes Toward Online Social Connection and Self-Disclosure as Predictors of Facebook Communication and Relational Closeness. Communication Research, $\mathrm{XX}(\mathrm{X}), 1-27$.

Lenhart, A., \& Madden, M. (2007). Teens, privacy and online social networks: How teens manage their online identities and personal information in the age of MySpace. Pew Internet \& American Life Project. Available at http://www.pewinternet.org/PPF/r/211/report_display.asp.

Lewis, J., \& West, A. (2009). 'Friending': London-based undergraduates' experience of Facebook. New Media \& Society, 11(7), $1209-1229$.

Licoppe, C. (2004). "Connected" Presence: The Emergence of a New Repertoire for Managing Social Relationships in a Changing Communication Technoscape. Environment and Planning D: Society and Space, 22(1), 135-56. 
Liu, H. (2007). Social network profiles as taste performances. Journal of Computer-Mediated Communication. Available at http://jcmc.indiana.edu/vol13/issue1/liu.html.

Livingstone, S. (2008). Taking Risky Opportunities in Youthful Content Creation: Teenagers' Use of Social Networking Sites for Intimacy, Privacy and Self-expression. New Media \& Society, 10(3), 393-411.

Macek, J. (2013). More than a desire for text: Online participation and the social curation of content. Convergence: The International Journal of Research into New Media Technologies, 19(3), 295-302.

Marsden, P. V. (2005). Recent developments in network measurement. In Carrington, P., Scott, J., Wasserman, S. (Eds.), Models and methods in social network analysis (pp. 8-30). Cambridge: Cambridge University Press.

Mazer, J. P., Murphy, R. E., \& Simonds, C. J. (2007). I'll see you on "Facebook": The effects of computer mediated teacher self-disclosure on student motivation, affective learning, and classroom climate. Communication Education, 56, 1-17.

McCarty, C. (2002). Structure in Personal Networks. Journal of Social Structure, 3(1). Available at http://www.cmu.edu/joss/content/articles/volindex.html.

Papacharissi, Z. (2009). The virtual geographies of social networks: a comparative analysis of Facebook, Linkedln and AsmallWorld. New Media Society, 11(1\&2), 199-220.

Pujazon-Zazik, M., \& Park, M. J. (2010). To Tweet, or Not to Tweet: Gender Differences and Potential Positive and Negative Health Outcomes of Adolescents' Social Internet Use. American Journal of Men's Health, 4(1), 77-85.

Ortiz-Arroyo, D. (2010). Discovering Sets of Key Players in Social Networks. În A. Abraham, A. Hassanien and V. Snasel (Eds.), Computational Social Network Analysis Trends, Tools and Research Advances (pp. 27-47). Springer-Verlag London Limited 2010.

Quan-Haase, A., \& Young, A. L. (2010). Uses and Gratifications of Social Media: A Comparison of Facebook and Instant Messaging. Bulletin of Science Technology \& Society, 30(5), 350 -361.

Schandorf, M. (2013). Mediated gesture: Paralinguistic communication and phatic text. Convergence: The International Journal of Research into New Media Technologies, 19(3), 319-344.

Scott, J. (2000). Social network analysis. A handbook. Sage: London.

Shepard, R. N. (1962). The analysis of proximities: Multidimensional scaling with an unknown distance function. Part 1. Psychometrika, 27, 125 - 140.

Smith, A., \& Rainie, L. (2008). The Internet and the 2008 election. Pew Internet \& American Life Project. Available at http://www.pewinternet.org/PPF/r/252/report_display.asp.

Stutzman, F. (2006). An Evaluation of Identity-sharing Behavior in Social Network Communities, paper presented at The Idmaa and IMS Code Conference, Oxford, OH, April 2006.

Tinnell, J. (2014). Computing en plein air: Augmented reality and impressionist aesthetics. Convergence: The International Journal of Research into New Media Technologies, 20(1), 69-84.

Tufekci, Z. (2008). Can You See Me Now? Audience and Disclosure Regulation in Online Social Network Sites. Bulletin of Science Technology \& Society, 28(1), 20-36.

Van Manen, M. (2010). The Pedagogy of Momus Technologies: Facebook, Privacy, and Online Intimacy. Qualitative Health Research, 20(8), 1023 - 1032.

Walther, J. B., van der Heide, B., Hamel, L. M., \& Shulman, H. C. (2009). Self-Generated Versus Other-Generated Statements and Impressions in Computer-Mediated Communication: A Test of Warranting Theory Using Facebook. Communication Research, 36(2), 229-253.

Watt, I. (2010). Changing visions of parliamentary libraries: From the Enlightenment to Facebook. International Federation of Library Associations and Institutions, 36(1), 47-60.

Wessels, B. (2012). Identification and the practices of identity and privacy in everyday digital communication. New Media \& Society, 14(8), 1251-1268.

Zhang, W., Johnson, T. J., Seltzer, T., \& Bichard, S. L. (2009). The Revolution Will Be Networked. The Influence of Social Networking Sites on Political Attitudes and Behavior. Social Science Computer Review, 28(1), 75-92. 


\section{APPENDIX}

Interview guide

How many friends do you have on Facebook now?

What is the social type of most of your friends? Do you consider that this aspect is irrelevant or did you have clear reasons for such a choice?

What age interval do most of your friends fall within?

What are your interests and hobbies (give at least two of the most relevant for each category)?

On what criteria do you initiate a friendship request or do you accept a friendship request on Facebook?

What category do most of your friends fall into (former faculty colleagues, faculty colleagues, offline close friends that you frequently interact with directly, neighbors from your native town or from the hostel, persons you know only online etc)? Is there any motivation for the main percentage of this category?

Which of your friends do you communicate most frequently and for the longest time on Facebook? What do you usually discuss upon?

What are, in your opinion, the advantages of communicating on Facebook as compared to communicating on other online social networks?

Have you ever joined a group because many of your friends were already part of the respective group? Why?

Do you consider that Facebook helps in developing relationships with any of the categories of friends? If yes, with whom the most and with whom the least? What arguments can you give to support your opinion?

What determines you to include in your list of friends a person that you have not yet met by direct interaction?

What determines you to communicate with this person frequently although you have not yet had the chance of meeting him / her directly?

What would be, according to you, the profile of a friend impossible to accept on Facebook? Please mention five essential attributes. 